\title{
Transmittance of transparent windows with non-absorbing cap-shaped droplets condensed on their backside
}

\author{
Keyong Zhu ${ }^{a}$, Yong Huang ${ }^{\mathrm{a}}$, Jeremy Pruvost ${ }^{\mathrm{b}}$, Jack Legrand ${ }^{\mathrm{b}}$, Laurent Pilon ${ }^{\mathrm{c}, *}$ \\ ${ }^{a}$ Beihang University School of Aeronautical Science and Engineering, No. 37, Xueyuan Road, Beijing 100191, China \\ ${ }^{\mathrm{b}}$ LUNAM Université, Université de Nantes, CNRS, GEPEA, UMR6144, Blvd de l'Université, CRTT - BP 406, 44602 Saint-Nazaire Cedex, France \\ ${ }^{\mathrm{C}}$ University of California, Los Angeles Mechanical and Aerospace Engineering Department, 420 Westwood Plaza, Los Angeles, CA 90095-1597, USA
}

\section{A R T I C L E I N F O}

\section{Article history:}

Received 14 December 2016

Received in revised form

8 February 2017

Accepted 6 March 2017

Available online 7 March 2017

Keywords:

Transmittance

Condensation

Windows

Greenhouses

Solar desalination

\begin{abstract}
A B S T R A C T
This study aims to quantify systematically the effect of non-absorbing cap-shaped droplets condensed on the backside of transparent windows on their directional-hemispherical transmittance and reflectance. Condensed water droplets have been blamed to reduce light transfer through windows in greenhouses, solar desalination plants, and photobioreactors. Here, the directional-hemispherical transmittance was predicted by Monte Carlo ray-tracing method. For the first time, both monodisperse and polydisperse droplets were considered, with contact angle between 0 and $180^{\circ}$, arranged either in an ordered hexagonal pattern or randomly distributed on the window backside with projected surface area coverage between 0 and $90 \%$. The directional-hemispherical transmittance was found to be independent of the size and spatial distributions of the droplets. Instead, it depended on (i) the incident angle, (ii) the optical properties of the window and droplets, and on (iii) the droplet contact angle and (iv) projected surface area coverage. In fact, the directional-hemispherical transmittance decreased with increasing incident angle. Four optical regimes were identified in the normal-hemispherical transmittance. It was nearly constant for droplet contact angles either smaller than the critical angle $\theta_{c r}$ (predicted by Snell's law) for total internal reflection at the droplet/air interface or larger than $180^{\circ}-\theta_{c r}$. However, between these critical contact angles, the normal-hemispherical transmittance decreased rapidly to reach a minimum at $90^{\circ}$ and increased rapidly with increasing contact angles up to $180^{\circ}-\theta_{c r}$. This was attributed to total internal reflection at the droplet/air interface which led to increasing reflectance. In addition, the normal-hemispherical transmittance increased slightly with increasing projected surface area coverage for contact angle was smaller than $\theta_{c r}$. However, it decreased monotonously with increasing droplet projected surface area coverage for contact angle larger than $\theta_{c r}$. These results can be used to select the material or surface coating with advantageous surface properties for applications when dropwise condensation may otherwise have a negative effect on light transmittance.
\end{abstract}

\section{Introduction}

The effects of water droplets condensate on the transmittance of plane parallel slabs or films of various semi-transparent materials (e.g., glass or plastic) have been investigated both experimentally and theoretically [1-16]. There exist two types of condensation namely (i) filmwise condensation referring to the formation of a liquid film covering the window and (ii) dropwise condensation corresponding to discrete droplets forming, growing, and potentially coalescing on the window [1]. Filmwise condensation on the backside of a window increases transmittance and reduces reflectance compared with a dry window thanks to

\footnotetext{
* Corresponding author.

E-mail address: pilon@seas.ucla.edu (L. Pilon).
}

the fact that the refractive index mismatch between air and water is smaller than between air and glass [16-19]. On the other hand, water droplets condensed on the backside of windows are known to decrease their transmittance because of backscattering and/or absorption of the incident radiation by the droplets $[1,9,10]$.

In practice, dropwise condensation reduces the solar energy input and the overall energy efficiency of greenhouses used for growing various plants [20]. In addition, droplet condensing inside greenhouses can cause damages and diseases to plants as they drip from the windows [6,7]. Dropwise condensation has also negative effects in other solar energy applications such as solar desalination where visible and infrared solar radiations are absorbed by salty water to evaporate freshwater which condenses and flows along an inclined windows before being collected [21]. The same is likely true in some microalgal culture systems, such as raceways or ponds covered by plastic sheets or glass plates [22] to 
grow microalgae and convert carbon dioxide and sunlight, in the photosynthetically active radiation (PAR) region $(400-700 \mathrm{~nm})$, into biomass.

Several approaches have been explored to favor the formation of filmwise rather than dropwise condensation. For example, anticondensation (or anti-drop) agents deposited on the window's backside can increase its wettability so that filmwise condensation prevailed to facilitate drainage of the condensate along the window surface [4-7]. Anti-dust measures can also be used to reduce static build-up on the window's outer surface by providing a conduction path to the ground [4].

This study aims to assess the effects of non-absorbing capshaped droplets condensed on the backside of transparent windows on their directional-hemispherical transmittance and reflectance. Particular attention was paid to the effects of light incident angle, as well as droplet size distribution, spatial arrangement, contact angle, and projected surface area coverage. The results will provide guidelines for material selection in order to minimize the effect of dropwise condensation on window transmittance.

\section{Background}

\subsection{Experiments}

Briscoe and Galvin [1] investigated experimentally the transport of monochromatic light at $650 \mathrm{~nm}$ through five types of glass windows with water droplets condensed on their backside. The windows consisted of $1.1 \mathrm{~mm}$ thick clean and unclean glass window as well as three glass windows coated, on their backside, with polymethyl methacrylate (PMMA or acrylic), polystyrene, and polyethylene (PE). The associated droplet contact angle was measured at $0^{\circ}, 50.9^{\circ}, 64.8^{\circ}, 90.0^{\circ}$, and $99.7^{\circ}$, respectively. In all cases, the surface area coverage of droplets was estimated to be $55 \%$, corresponding to the equilibrium coverage accounting for droplet coalescence on a vertical substrate [23-25]. The authors measured the normal-hemispherical reflectance of each window held vertically under dry or dropwise condensation conditions. The normalhemispherical reflectance $R_{n h, 650}$ of the window with droplet condensation was found to increase from about $5 \%$ to $24 \%$ as the contact angle increased from about $0^{\circ}$ to $100^{\circ}$. Simultaneously, the normal-hemispherical transmittance $T_{n h, 650}$ decreased with increasing contact angle. Note that absorption by the glass window and the polymeric films can be neglected at the wavelength considered, so that $T_{n h, 650}=1-R_{n h, 650}$.

Pollet and Pieters [4] measured the directional-hemispherical transmittance of visible light at $632.8 \mathrm{~nm}$ through three types of vertical glass plates namely (i) a $4 \mathrm{~mm}$ thick single glass plate, (ii) a $4 \mathrm{~mm}$ thick single glass plate with a low-emissivity $\mathrm{SnO}_{2}$ coating, and (iii) a $8 \mathrm{~mm}$ thick double glass plate, along with three types of vertical polyethelene films including (iv) a $150 \mu \mathrm{m}$ thick singlelayer ordinary low-density PE film, (v) a $180 \mu \mathrm{m}$ thick three-layer anti-drop polyvinyl-ethyl vinyl acetate (PE-EVA) film, and (vi) a $180 \mu \mathrm{m}$ thick three-layer anti-dust PE-EVA film. The authors reported that condensed water droplets reduced the directionalhemispherical transmittance of all glass plates and PE-based films for light incident angles ranging from 0 (normal) to $75^{\circ}$. For example, the transmittance of the low-density PE film decreased by up to $23 \%$ under normal incidence due to the presence of droplets. However, the directional-hemispherical transmittance of the antidrop PE-EVA film was not affected by water condensation and featured the largest transmittance under condensation conditions. The authors attributed the different effects of dropwise condensation on the directional-hemispherical transmittance to the shape of droplets which were much flatter on the glass windows than on the PE films. They also observed wide variations in the shape, size, and spatial distributions of the droplets condensed on the glass plates and PE films. Droplets condensed on the PE films without anti-drop agents approached more closely the cap-shaped than those on the glass slabs. However, the authors did not measure either the contact angle or the surface area coverage of the droplets because of their irregular shapes.

Geoola et al. [6,7] designed an experimental greenhouse and measured the total hemispherical transmittance of solar radiation (collimated and diffuse) over the wavelength range 305-2800 nm through $140 \mu \mathrm{m}$ thick cladding materials with and without condensation on the inner surface. Three types of low-density PE films were investigated namely (i) ultraviolet (UV)-stabilized PE films, (ii) UV- stabilized and infrared-modified PE films, and (iii) ultraviolet-stabilized as well as infrared- and anti-drop-modified PE films. The total hemispherical transmittance of the anti-drop PE film was larger under condensation conditions by about 3.5\% than under dry conditions. By contrast, the total hemispherical transmittance of the two other types of PE films without anti-drop agents was lower by about 14-19\% between dropwise condensation and dry conditions. Unfortunately, the authors did not report the contact angle and surface area coverage of the droplets condensed on the different PE films.

Cemek and Demir [8] investigated experimentally the total hemispherical transmittance of solar radiation in mini-greenhouses with and without dropwise condensation on the inner surface. Four kinds of covering materials were tested namely (i) PE films with no additives, (ii) UV-stabilized PE films, (iii) IR-absorbing PE films, and (iv) double-layer PE films with no additives, all with a thickness of $150 \mu \mathrm{m}$. The authors measured the number and diameter of droplets on the oblique ceiling and vertical sidewall of the greenhouses and calculated the volume and surface area coverage of droplets assumed to be cap-shaped. The mean droplet diameter on the greenhouse's ceiling made of PE films with no additives, UV-stabilized PE films, and IR-absorbing PE films were $2.6 \mathrm{~mm}, 2.0 \mathrm{~mm}$, and $2.6 \mathrm{~mm}$, respectively with surface area coverage of $46 \%, 29 \%$, and $38 \%$. Droplets diameter on the greenhouse vertical sidewalls, for the same three PE films, were $1.2 \mathrm{~mm}, 1.2 \mathrm{~mm}$, and $2.6 \mathrm{~mm}$ with surface area coverage of $48 \%$, $16 \%$, and $23 \%$, respectively. The total hemispherical transmittance of solar radiation was larger by about $9 \%$ under dry conditions compared with condensation conditions for all types of PE films. The total hemispherical transmittance of double-layer PE films was the lowest while that of PE films with no additives was the highest both under dry and condensation conditions. The authors indicated that high surface area coverage and volume of condensate led to a reduction of the total hemispherical transmittance.

Overall, previous experimental measurements have established that water droplet condensation on the backside of windows decrease their directional-hemispherical transmittance, compared with dry conditions except for some anti-drop films [1,4,6-8]. In addition, it was also established that the normal-hemispherical transmittance decreases with increasing contact angle. Only contact angles up to $90^{\circ}-100^{\circ}$ were explored [1]. To the best of our knowledge, window materials with large contact angles were not considered.

\subsection{Modeling}

Hsieh and Rajvanshi [9] analyzed theoretically light transfer by the ray-tracing method at wavelengths between $500 \mathrm{~nm}$ and $2.5 \mu \mathrm{m}$ for a $2.286 \mathrm{~mm}$ thick absorbing glass slab supporting, on its backside, a single hemispherical water droplet, i.e., with contact angle of $90^{\circ}$. The droplet radius varied from $0.25 \mathrm{~mm}$ to $1.25 \mathrm{~mm}$. The droplet size was found to have a negligible effect on both 
spectral normal-hemispherical transmittance and reflectance at wavelengths below $900 \mathrm{~nm}$ because of the low absorption coefficient of both glass and water. However, both spectral normalhemispherical transmittance and reflectance decreased with increasing droplet size and wavelength beyond $900 \mathrm{~nm}$. Then, the authors expressed the normal-hemispherical transmittance $T_{n h, \lambda}$ of glass windows supporting multiple droplets as the sum of the normal-hemispherical transmittances of the dry window $T_{n h, \lambda, 0}$ and of the wet window with a single droplet $T_{n h, \lambda, 1}$ weighted by the fraction $f_{\mathrm{A}}$ of window surface area covered by the projected droplets according to $[1,9]$

$T_{n h, \lambda}=f_{\mathrm{A}} T_{n h, \lambda, 1}+\left(1-f_{\mathrm{A}}\right) T_{n h, \lambda, 0}$.

Briscoe and Galvin [1] presented a ray-tracing analysis to predict the normal-hemispherical transmittance at $650 \mathrm{~nm}$ through a glass slab $\left(n_{w}=1.5\right)$ supporting a single cap-shaped water droplet $\left(n_{d}=1.33\right)$ condensed on its backside with contact angle ranging from $0^{\circ}$ to $120^{\circ}$. The thickness of the glass slab was $1.1 \mathrm{~mm}$ and the diameter of the droplet was $50 \mu \mathrm{m}$. The absorption coefficients $\kappa_{w}$ of the glass window and $\kappa_{d}$ of water droplets at $650 \mathrm{~nm}$ were both assumed to be $0.04 \mathrm{~mm}^{-1}$. The normal-hemispherical reflectance was found to be independent of contact angle up to about $40^{\circ}$ and increased rapidly beyond. This was attributed to total internal reflection at the water-air interface. The authors also adopted the surface area coverage-weighted sum of the normal-hemispherical reflectances of the glass slab without and with a single cap-shaped droplet for surface area coverage estimated at 55\%, as previously discussed. Good quantitative agreement was obtained between simulation predictions and experimental measurements of the normal-hemispherical reflectance. Note, however, that the absorption coefficient $\kappa_{\lambda}=4 \pi k_{\lambda} / \lambda$ of clear soda-lime silicate glass and water at wavelength $\lambda$ of $650 \mathrm{~nm}$ can be estimated as $0.016 \mathrm{~mm}^{-1}$ [26] and $3.2 \times 10^{-4} \mathrm{~mm}^{-1}$ [27], respectively. These values are significantly different from those used in the simulations [1].

Pieters et al. [10] also simulated light transfer by the ray-tracing method at the wavelength of $632.8 \mathrm{~nm}$ through an absorbing glass slab $\left(n_{w}=1.526, \kappa_{w}=4 \mathrm{~m}^{-1}\right)$ or a polyethylene film $\left(n_{w}=1.515\right.$, $\left.\kappa_{w}=165 \mathrm{~m}^{-1}\right)$ with a single absorbing cap-shaped droplet $\left(n_{d}=\right.$ $1.515, \kappa_{d}=0.4 \mathrm{~m}^{-1}$ ) on their backside with contact angle ranging from $10^{\circ}$ to $90^{\circ}$. The droplet radius, ranging from $1.275 \mathrm{~mm}$ to $12.75 \mathrm{~mm}$, was found to have no effect on the transmittance. However, the directional-hemispherical transmittance decreased with increasing droplet contact angle from $10^{\circ}$ to $90^{\circ}$. In addition, Pieters [11] further improved the previous model by simulating light transfer through a slab or film supporting two adjacent droplets. The author indicated that the directional-hemispherical transmittance of a window partly covered with droplets was different from the surface area coverage-weighted sum of the wet and dry transmittances, given by Eq. (1) [1,9], especially for large incident angle due to potential light reflection and refraction from one droplet to another directly or via the glass slab.

Tow [15] investigated the directional-hemispherical reflectance by the ray-tracing method at wavelength $500 \mathrm{~nm}$ for non-absorbing monodisperse cap-shaped water droplets $\left(n_{d}=1.33, k_{d}=0\right)$ in a hexagonal arrangement on the backside of a non-absorbing glass window ( $\left.n_{w}=1.5, k_{w}=0\right)$ as a function of (i) contact angle ranging from $0^{\circ}$ to $90^{\circ}$, (ii) incident angle between $0^{\circ}$ and $80^{\circ}$, and (iii) droplet surface area coverage up to $90 \%$. The glass window was $3 \mathrm{~mm}$ thick and the monodisperse droplets were $2.66 \mathrm{~mm}$ in diameter. The normal-hemispherical reflectance was found to increase with increasing surface area coverage for contact angle of $60^{\circ}$ but decreased with increasing surface area coverage for contact angle of $20^{\circ}$ and $40^{\circ}$. The author also suggested that condensation of water droplets could act as an optically antireflective coating as droplets condensation could

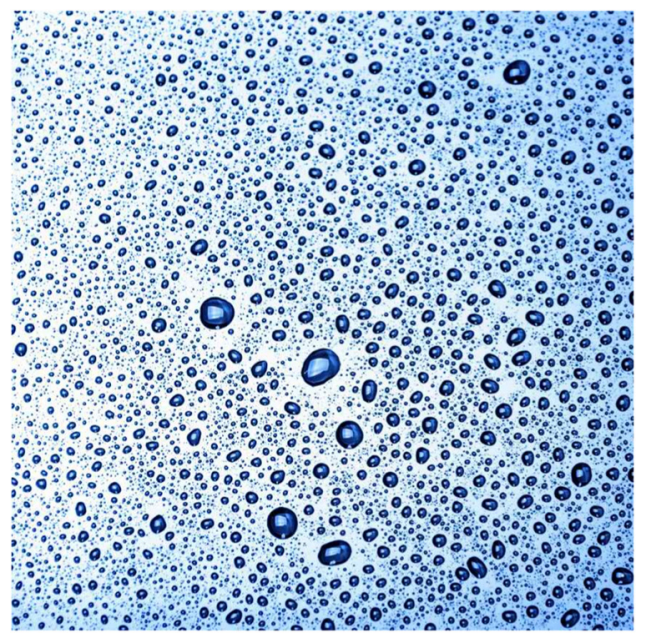

Fig. 1. Photograph of a glass window with randomly distributed and polydisperse condensed water droplets.

decrease the normal-hemispherical reflectance of glass to below that of glass coated with a water film when the contact angle ranges between $26^{\circ}$ and $49^{\circ}$ with $90 \%$ surface area fraction.

Most previous theoretical studies have simulated light transfer through a glass slab or plastic film supporting a single and several monodisperse and ordered cap-shaped droplets. They qualitative confirmed experimental observations that the directional-hemispherical transmittance decreased with the presence of droplets and with increasing contact angle up to $120^{\circ}[1,10]$. In practice, however, condensed droplets are numerous, polydisperse, and randomly distributed on the window's surface, as illustrated in Fig. 1. Moreover, the window can be made of a wide range of materials with different complex index of refraction and wettability. The present study aims to systematically investigate numerically light transfer through transparent windows with different refractive index supporting numerous non-absorbing monodisperse or polydisperse condensed droplets ordered or randomly distributed on the substrate's backside with contact angle between 0 and $180^{\circ}$.

\section{Analysis}

\subsection{Problem statement}

Figs. 2(a) and (b) show the top and side views of the simulated polydisperse droplets randomly distributed on a window with length $L$, width $W$, and thickness $H$. The window was exposed to collimated radiation incident on its front side at a polar angle $\theta_{i}$. Photons were reflected and refracted by the transparent window of refractive index $n_{w}$. Part of the incident radiation was transmitted through the back face of the window into the non-absorbing droplets, with refractive index $n_{d}$. Then, radiation could be internally reflected within the droplet or transmitted through the droplet/air or the droplet/window interfaces. In the present study, the dimensions of the window for simulating randomly distributed and monodisperse or polydisperse droplets were $L=W$ $=5 \mathrm{~mm}$, and $H=3 \mathrm{~mm}$. The refractive indices of air and water in the PAR region were taken as $n_{a}=1.0$ and $n_{d}=1.33$ [27], respectively. The window was assumed to be glass with refractive index of $n_{w}=1.5$ [26]. The boundary conditions on the sides of the window were periodic. 
(a)

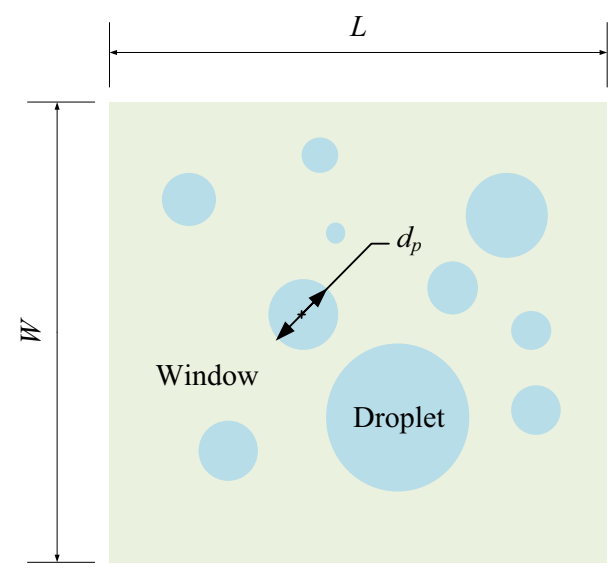

(b)

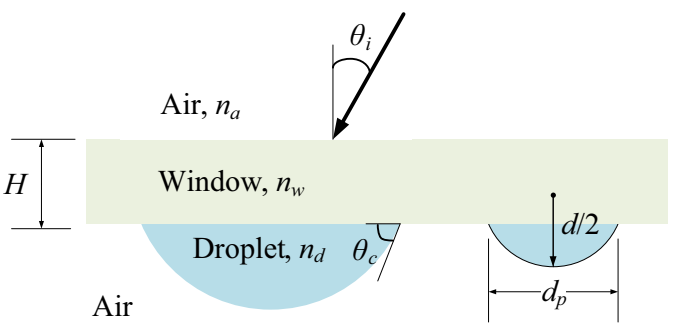

Fig. 2. (a) Top view of the transparent window supporting polydisperse droplets. (b) Cross-section of the transparent window supporting non- absorbing droplets.

\subsection{Droplet generation}

In the present study, three droplet configurations with different size distributions and spatial arrangements on the window surface were considered namely (i) monodisperse droplets ordered in a hexagonal pattern, (ii) randomly distributed monodisperse droplets, and (iii) randomly distributed polydisperse droplets with a normal size distribution.

Droplets deposited on a flat surface assume a cap-shaped form with constant curvature if their diameter $d$ is much smaller than the capillary length $l_{c}$ defined as [28]

$l_{c}=\sqrt{\frac{\gamma}{\rho g}}$

where $\gamma$ is the surface tension of the droplet/air interface, $\rho$ is the droplet density, and $g$ is the gravitational acceleration $(g=9.81 \mathrm{~m} /$ $\mathrm{s}^{2}$ ). For water droplets in air, $\gamma=72.1 \mathrm{mN} / \mathrm{m}$ and $\rho=1000 \mathrm{~kg} / \mathrm{m}^{3}$ so that $l_{c}=2.7 \mathrm{~mm}$.

The procedure to generate droplets randomly distributed on the window surface with given (i) size distribution with mean droplet diameter $d_{m}$ and standard deviation $\sigma$ and (ii) projected surface area coverage $f_{\mathrm{A}}$ followed three steps. Step 1 consisted of randomly generating droplet diameter $d$ according to

$d=d_{m}+R_{d} \sigma$

where $d_{m}$ and $\sigma$ are the imposed average diameter and standard deviation of the droplet diameter normal distribution while $R_{d}$ is a random number of standard normal distribution with a mean of 0 and standard deviation equal to 1 . Note that the projected droplet diameter $d_{p}$ can be measured more easily than $d$ and is simply expressed as

$d_{p}=d \sin \theta_{c} \quad$ for $\theta_{c}<90^{\circ} \quad$ and $\quad d_{p}=d$ for $\theta_{c} \geq 90^{\circ}$
In addition, the droplet diameter $d$ satisfied $d_{m}-3 \sigma \leq d \leq d_{m}+3 \sigma$ and was such that $0<d<l_{c} / 10$. If it fell outside this interval, it was rejected and another diameter was generated. This was repeated for a total number of $N$ droplets until the projected surface area coverage $f_{\mathrm{A}}$ reached the desired value. Step 2 consists of sorting the droplets generated in Step 1 by decreasing diameter with droplet index $i$ varying from 1 to $N$. In Step 3, the positions $\left(x_{i}\right.$, $\left.y_{i}\right)_{1 \leq i \leq N}$ of the centers of the discs corresponding to the projection of the cap-shaped droplet onto the window's surface were generated randomly. The droplets were assigned to their random location $\left(x_{i}, y_{i}\right)_{1 \leq i \leq N}$ by decreasing diameter, as sorted in Step 2. If a droplet overlapped with an already placed droplet, the location was rejected and another random center position was generated until the droplet did not overlap with any other one.

Fig. 3 shows examples of the projected circles of droplets of a $5 \mathrm{~mm} \times 5 \mathrm{~mm}$ window section. The randomly distributed and polydisperse droplets were generated with mean diameter $d_{m}=$ $100 \mu \mathrm{m}$ and standard deviation $\sigma=30 \mu \mathrm{m}$, with $\theta_{c}=90^{\circ}$ for projected surface area coverage $f_{\mathrm{A}}$ equals to (a) $20 \%$, (b) $30 \%$, and (c) $40 \%$ corresponding, in these specific illustrations, to a total number of droplets equal to 378,563 , and 757 , respectively.

\subsection{Light transmission}

\subsubsection{Assumptions}

Several assumptions were made in our Monte Carlo ray-tracing simulations:

1) All interfaces were optically smooth so that all reflections were specular and Snell's law and Fresnel's equations were valid [29] (see Supplementary Material).

2) The droplets were cap-shaped with a constant curvature and their diameter was smaller than $270 \mu \mathrm{m}$ to satisfy $d \ll l_{c}$.

3) The window and droplets were non-absorbing and had a constant and uniform refractive index at the wavelengths of interest.

4) The dimensions of the droplets and window were much larger than the wavelength so geometric optics prevailed and interferences and other wave effects could be ignored.

5) Potential precursor films near the droplets' edges have characteristic dimensions much smaller than the radiation wavelength $[30,31]$ and can be ignored in our analysis.

\subsubsection{Monte Carlo ray-tracing method}

Monte Carlo ray-tracing method [32] was used to predict the directional-hemispherical transmittance of a transparent window exposed to collimated radiation and supporting condensed non-absorbing cap-shaped droplets on its backside, generated by the methodology previously described. The incident photon bundles may experience multiple reflections and refractions at the window/air, window/droplet, and droplet/air interfaces. They were traced until they reached either the imaginary surface above the dry surface of the window, when they were counted as reflected, or the imaginary surface beyond the droplets, when they were counted as transmitted. The computational steps were as follows for each photon bundle (see block diagram in Fig. S2 of Supplementary Material):

1) For a given incident angle $\theta_{i}$, determine the random position vector $\mathbf{p}_{0}=\left(x_{0}, y_{0}\right)$ in the $\mathrm{x}$-y plane of the frontside (dry) of the window where the incident photons reached the window.

2) Determine the random incident direction $\mathbf{s}_{i}$ defined by a specific polar angle $\theta_{i}$ and a random azimuthal angle $\varphi_{i}$.

3) Calculate the reflectance $\rho$ of the air/window interface using Fresnel's equations. Generate a random number between 0 and 1 obeying uniform distribution. If the random number is smaller than $\rho$, then reflection occurs, otherwise, refraction occurs. 

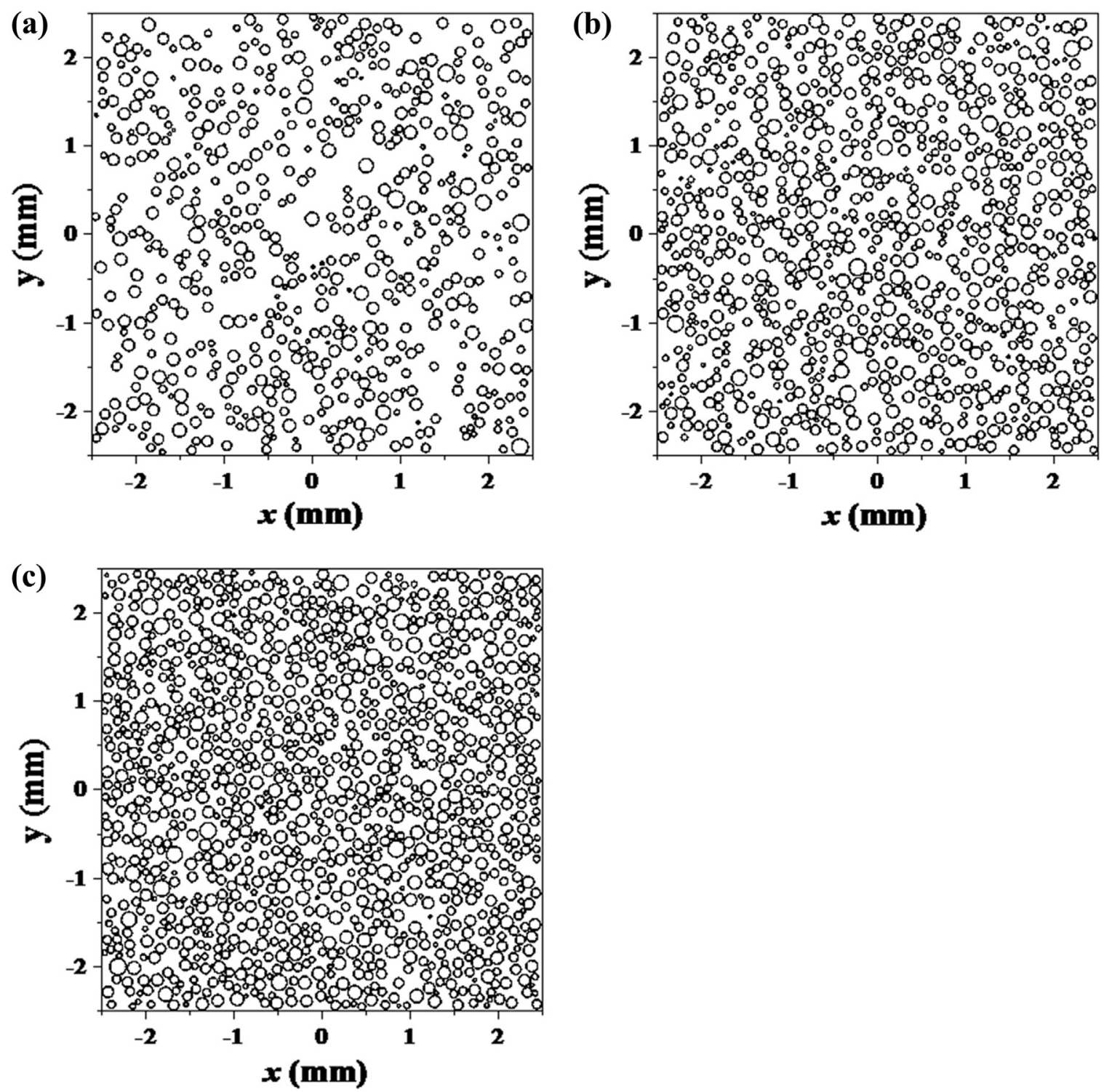

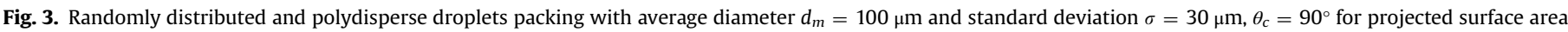
coverage $f_{\mathrm{A}}$. (a) $f_{\mathrm{A}}=20 \%$ with $N=592$ droplets, (b) $f_{\mathrm{A}}=30 \%$ with $N=902$ droplets, and (c) $f_{\mathrm{A}}=40 \%$ with $N=1178$ droplets.

4) Determine the specular reflection and refraction directions $\mathbf{s}_{r}$ or $\mathbf{s}_{t}$ from Snell's law.

5) Calculate the position $\mathbf{p}^{\prime}$ of the next interface reached by the photon bundle based on geometric considerations.

6) Reset the initial location to $\mathbf{p}_{0}=\mathbf{p}^{\prime}$ and directions $\mathbf{s}_{\mathrm{i}}=\mathbf{s}_{\mathrm{r}}$ or $\mathbf{s}_{\mathrm{i}}=\mathbf{s}_{\mathrm{t}}$. Then repeat Steps 3 to 5 .

Whenever the updated position $\mathbf{p}_{0}$ is above the dry front side of the window, it indicates that the photon is reflected. Then the number of reflected photons $M_{R}$ is incremented, i.e., $M_{R}=M_{R}+1$. Similarly, if the position $\mathbf{p}_{0}$ is beyond all the droplets, it means that the photon is transmitted. Then, the number of photons $M_{T}$ is incremented, i.e., $M_{T}=M_{T}+1$. The ray-tracing procedure ends for the traced photon bundle and the procedure restarts for a new photon bundle at Step 1.

Finally, after tracing $M$ statistically significant number of photon bundles, the directional-hemispherical reflectance $R_{d h}$ and transmittance $T_{d h}$ of the wet window are calculated according to

$R_{d h}=M_{R} / M \quad$ and $\quad T_{d h}=M_{T} / M$

Here, $M=M_{R}+M_{T}$ or $R_{d h}+T_{d h}=1$ since the window and the droplets are non-absorbing. In all simulation results reported in this study, the total number of photon bundles simulated was $M=10^{6}$ in order to achieve numerically converged results independent of the number of photon bundles simulated (see Fig. S3 in Supplementary Material).

\subsubsection{Validation}

In order to validate the Monte Carlo ray-tracing procedure and algorithm, we considered the case of monodisperse water droplets $\left(n_{d}=\right.$ 1.33) of diameter $d=100 \mu \mathrm{m}$ ordered in a hexagonal pattern on the backside of a glass window $\left(n_{w}=1.5\right)$ of thickness $H=3 \mathrm{~mm}$, as illustrated in Fig. 4. First, we simulated light transfer through a glass window supporting eight water droplets and using periodic boundary conditions [Fig. 4(a)]. By virtue of symmetry, this configuration should be equivalent to a triangular slice corresponding to 1/12th of a hexagon with a single droplet with reflecting boundary conditions [Fig. 4(b)]. Similar simulations were performed by Tow [15]. Excellent agreement was found between the two approaches confirming the validity of the algorithms and proper consideration of the optical phenomena and boundary conditions (see Fig. S4 in Supplementary Material). The present results were also consistent with numerical results reported in 
(a)

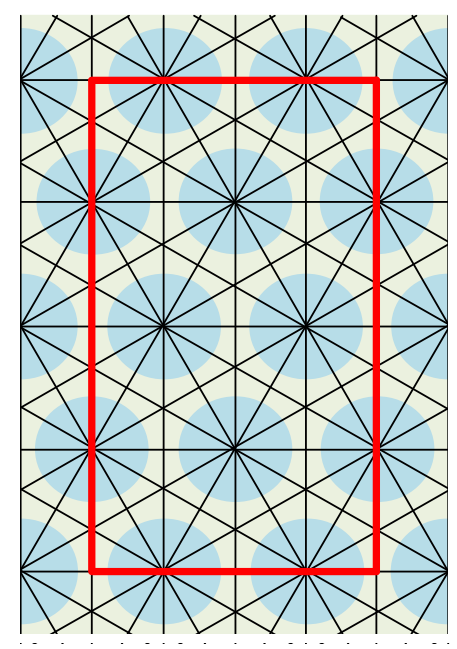

(b)

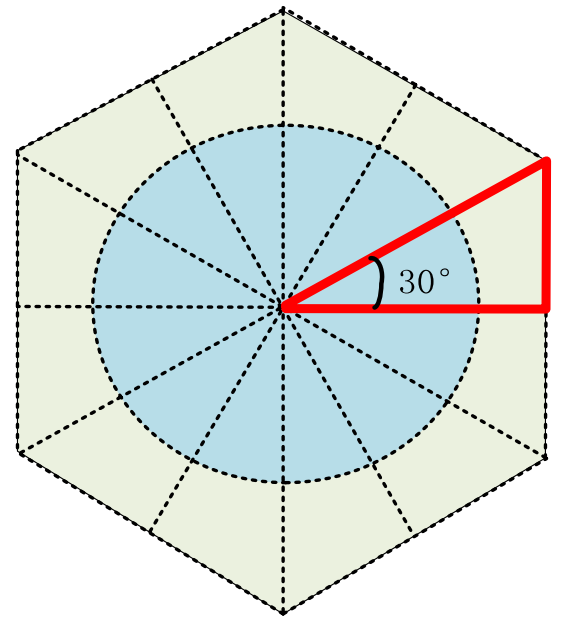

Fig. 4. Simulated geometries for monodisperse droplets ordered in a hexagonal pattern with $d=100 \mu \mathrm{m}$. (a) section of eight droplets with periodic boundary conditions and (b) triangular slice corresponding to $1 / 12$ th of a hexagon with reflecting boundary conditions.

the literature [1] (see Fig. S5 in Supplementary Material).

\section{Results and discussion}

In this study, the effects of (i) incident angle as well as (ii) droplet's spatial distribution, (iii) size distribution, (iv) contact angle, and (v) projected surface area coverage on the directional-hemispherical transmittance of transparent windows with non-absorbing water droplets condensing on their backside were systematically investigated over a wide range of parameters. Note that the thickness of the glass window was found to have no effect on the directionalhemispherical transmittance by virtue of the fact that it was nonabsorbing (see Fig. S6 in Supplementary Material).

\subsection{Effect of droplet size and spatial distributions}

Fig. 5 shows the directional-hemispherical transmittance of glass windows with condensed water droplets as a function (a) of incident angle $\theta_{i}$ for contact angle $\theta_{c}=60^{\circ}$ and projected surface area coverage $f_{\mathrm{A}}=30 \%$ and (b) of contact angle $\theta_{c}$ between 0 and $180^{\circ}$ under normal incident (i.e., $\theta_{i}=0^{\circ}$ ) and $f_{\mathrm{A}}=30 \%$. Each plot compares the predictions of the directional-hemispherical transmittance for four configurations namely (i) monodisperse droplets of diameter $d_{m}=100 \mu \mathrm{m}$ arranged in an ordered hexagonal (a)

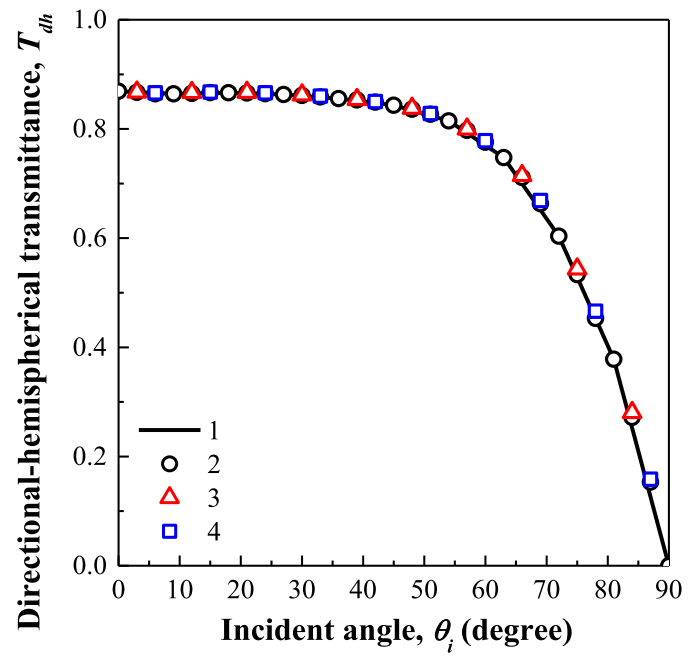

(b)

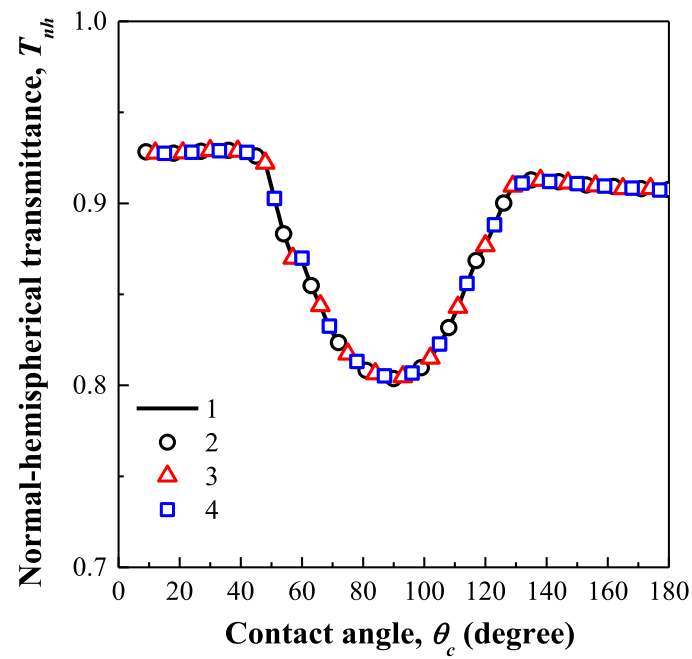

Fig. 5. Directional-hemispherical transmittance: (a) as a function of incident angle $\theta_{i}$ with $\theta_{c}=60^{\circ}$ and $f_{\mathrm{A}}=30 \%$ and (b) as a function of contact angle $\theta_{c}$ with $\theta_{i}=0^{\circ}$ and $f_{\mathrm{A}}=30 \%$ for four different configurations. 1. Monodisperse droplets of diameter $d_{m}=100 \mu \mathrm{m}$ arranged in an ordered hexagonal pattern. 2. Monodisperse and randomly distributed droplets of diameter $d_{m}=100 \mu \mathrm{m}$. 3. Polydisperse and randomly distributed droplets with $d_{m}=100 \mu \mathrm{m}$ and $\sigma=30 \mu \mathrm{m}$. 4. Polydisperse and randomly distributed droplets $d_{m}=200 \mu \mathrm{m}$ and $\sigma=60 \mu \mathrm{m}$.

pattern, (ii) monodisperse and randomly distributed droplets of diameter $d_{m}=100 \mu \mathrm{m}$, as well as (iii) polydisperse and randomly distributed droplets following a normal distribution with mean diameter $d_{m}=100 \mu \mathrm{m}$ and standard deviation $\sigma=30 \mu \mathrm{m}$, and (iv) $d_{m}=200 \mu \mathrm{m}$ and $\sigma=60 \mu \mathrm{m}$. To the best of our knowledge, Fig. 5 reports the first simulations of such a large number of randomly distributed polydisperse droplets with contact angles above $120^{\circ}$. Fig. 5(a) indicates that the directional-hemispherical transmittance was nearly constant for incident angle $\theta_{i}$ between $0^{\circ}$ and $60^{\circ}$ beyond which it decreased sharply to vanish at $90^{\circ}$.

Moreover, Figs. 5(a) and (b) indicate that the directionalhemispherical transmittance was independent of the droplet spatial arrangement (i.e., ordered or random) and of the droplet size distribution (monodisperse or polydisperse). Additionally, the normal-hemispherical transmittance was also predicted for wet glass window supporting polydisperse droplets of different size distributions namely (i) normal distribution, (ii) uniform distribution, and (iii) gamma distribution. Results indicate that the size distribution had no effect on the normal-hemispherical transmittance albeit for a given projected surface area coverage $f_{\mathrm{A}}$ (see Fig. S7 in Supplementary Material). These results corroborate 
(a)

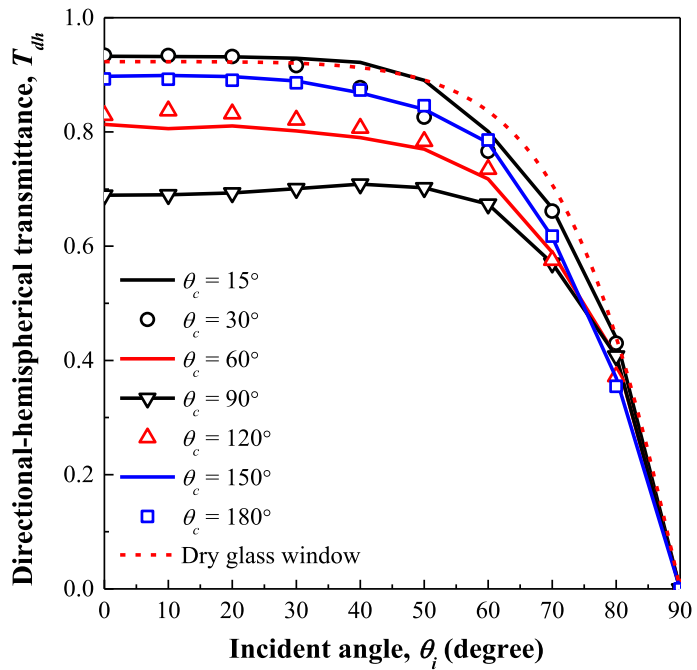

(b)

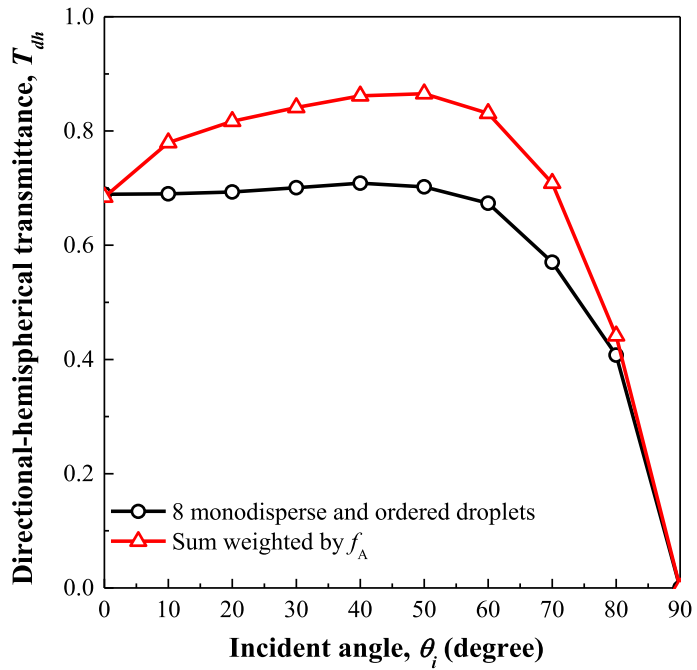

Fig. 6. Directional-hemispherical transmittance as a function of incident angle $\theta_{i}$ (a) for different values of contact angle $\theta_{c}$ and $f_{\mathrm{A}}=60 \%$ and (b) for $\theta_{c}=90^{\circ}$ and $f_{\mathrm{A}}$ $=60 \%$, where the black line was the transmittance in the model of monodisperse and ordered droplets, while the red line was the transmittance predicted by Eq. (1).

previous numerical studies which established that, for a single droplet with a given projected surface area coverage, the droplet diameter had a negligible effect on the normal-hemispherical transmittance and reflectance $[1,9,10]$. The present findings reduce significantly the number of parameters on which the directionalhemispherical transmittance depends, i.e., $T_{d h}=T_{d h}\left(\theta_{i}, n_{w}, n_{d}, \theta_{c}\right.$, $f_{A}$ ). In fact, results presented in the remaining of this study, were obtained for monodisperse droplets of diameter $d_{m}=100 \mu \mathrm{m}$ ordered in a hexagonal pattern.

\subsection{Effect of incident angle}

Fig. 6(a) plots the directional-hemispherical transmittance as a function of incident angle $\theta_{i}$ for droplet projected surface area coverage of $f_{\mathrm{A}}=60 \%$ and contact angle $\theta_{c}$ of $15^{\circ}, 30^{\circ}, 60^{\circ}, 90^{\circ}, 120^{\circ}, 150^{\circ}$, and $180^{\circ}$, and for a dry glass window. The directional-hemispherical transmittance decreased with increasing contact angles from $15^{\circ}$ to $90^{\circ}$. However, it increased with increasing contact angles from $90^{\circ}$ to $180^{\circ}$, for all incident angles. It decreased monotonously with incident angle $\theta_{i}$ for contact angles $15^{\circ}, 30^{\circ}, 60^{\circ}, 120^{\circ}, 150^{\circ}$ and $180^{\circ}$. However, for contact angle $\theta_{c}=90^{\circ}$, the directional-hemispherical transmittance (a)

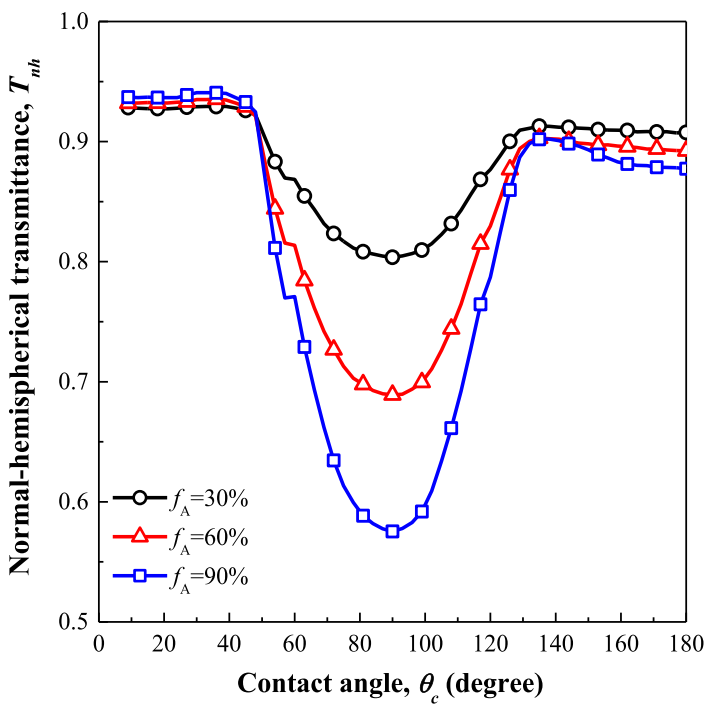

(b)

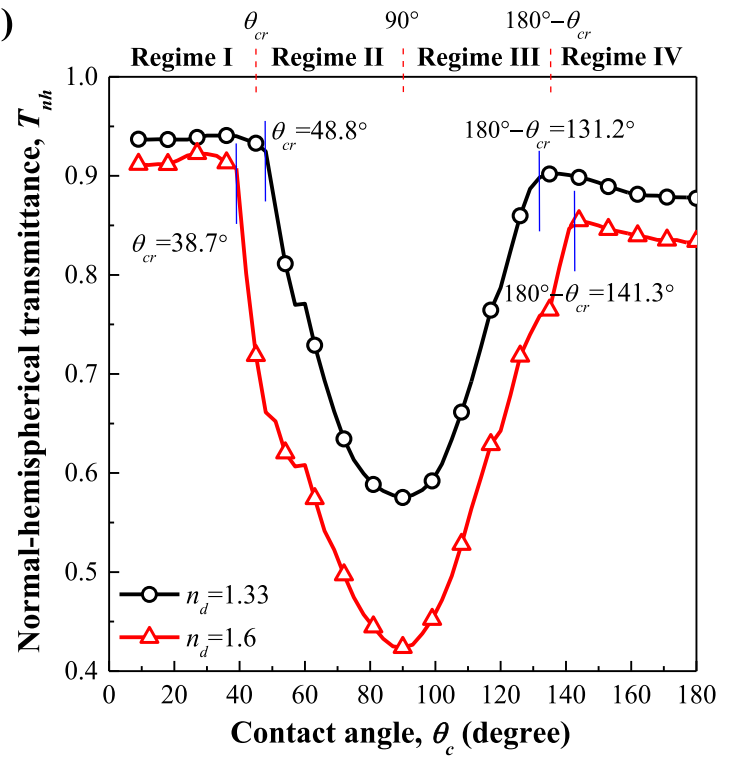

Fig. 7. Normal-hemispherical transmittance as a function of contact angle $\theta_{c}$ (a) for different values of projected surface area coverage $f_{\mathrm{A}}$ and (b) for different droplet refractive index $n_{d}$ and critical angle $\theta_{c r}$, at the liquid-air interface for $f_{\mathrm{A}}=90 \%$.

feature a maximum for incident angle $\theta_{i}$ around $50^{\circ}$, as also observed in Ref. [10]. Furthermore, the directional-hemispherical transmittance for $\theta_{c}=60^{\circ}, 90^{\circ}, 120^{\circ}, 150^{\circ}, 180^{\circ}$ was smaller than that of the dry glass window for all incident angles. However, the directional-hemispherical transmittance for $\theta_{c}=15^{\circ}$ and $30^{\circ}$ was slightly larger than that of the dry glass window for incident angle $\theta_{i} \leq 45^{\circ}$ with $\theta_{c}=15^{\circ}$ and $\theta_{i} \leq$ $25^{\circ}$ with $\theta_{c}=30^{\circ}$.

Fig. 6(b) compares the directional-hemispherical transmittance as a function of incident angle for wet glass window with $\theta_{c}=90^{\circ}$ and $f_{\mathrm{A}}=60 \%$ (i) predicted numerically by the Monte Carlo ray-tracing method and (ii) predicted as the projected surface area coverageweighted sum of transmittances for a dry and a wet window given by Eq. (1).

The two approaches gave similar results for normal incidence, i.e., $\theta_{i}=0^{\circ}$. However, for non-zero incident angles, predictions by Eq. (1) significantly underpredicted the transmittance $T_{d h}$ predicted by the Monte Carlo ray-tracing method for multiple droplets. This could be attributed to the fact that photons with large incident angle may travel through multiple droplets with increasing likelihood of being reflected before being transmitted. 
(a) Regime I: $\theta_{c}<\theta_{c r}$

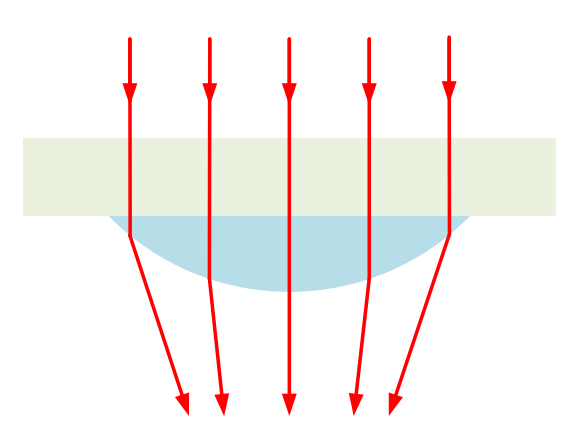

(c) Regime III: $90^{\circ} \leq \theta_{c}<180^{\circ}-\theta_{c r}$

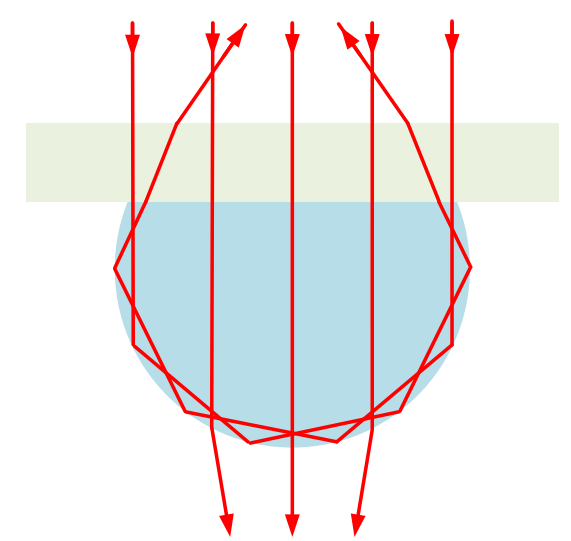

(b) Regime II: $\theta_{c r} \leq \theta_{c}<90^{\circ}$

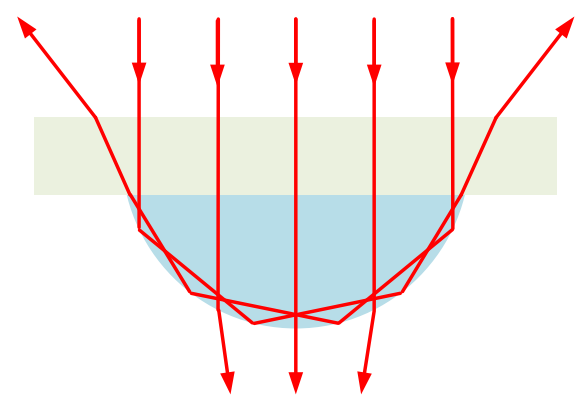

(d) Regime IV: $\theta_{c} \geq 180^{\circ}-\theta_{c r}$

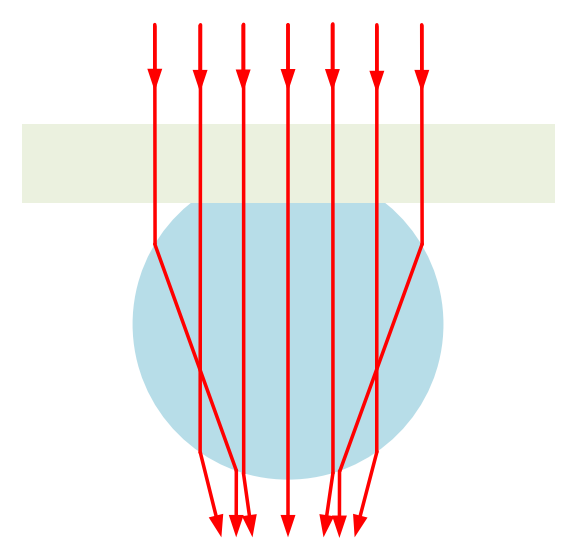

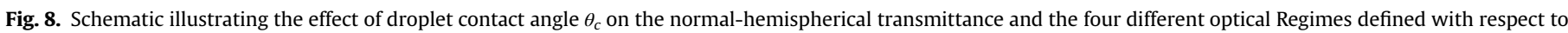
total internal reflection with critical angle $\theta_{c r}=\sin ^{-1}\left(1 / n_{d}\right)$.

This would decrease the directional-hemispherical transmittance, as suggested in Ref. [11] from simulations of two adjacent droplets of identical diameter.

\subsection{Effect of contact angle and critical angle}

This section pays particular attention to the effect of contact angle and critical angle on the normal-hemispherical transmittance. Indeed, the directional-hemispherical transmittance was nearly unchanged for incident angle between 0 and $60^{\circ}$ and rapidly decreased to vanish at $90^{\circ}$. Thus, the normal-hemispherical is representative of the total transmittance and energy flux passing through the window supporting condensed droplets.

Fig. 7(a) plots the normal-hemispherical transmittance $T_{n h}$ as a function of droplet contact angle $\theta_{c}$ between $0^{\circ}$ and $180^{\circ}$ for droplet refraction index $n_{d}=1.33$ and projected surface area coverage $f_{\mathrm{A}}$ equal to 30,60 , and $90 \%$. Similarly, Fig. 7(b) plots $T_{n h}$ for projected surface area coverage $f_{\mathrm{A}}$ of $90 \%$ and different values of droplet refractive index $n_{d}$ namely 1.33 and 1.6 corresponding to critical angle $\theta_{c r}=\sin ^{-1}\left(1 / n_{d}\right)$ for total internal reflection at the droplet/air interface of $48.8^{\circ}$ and $38.7^{\circ}$, respectively. Fig. 7 indicates that the normalhemispherical transmittance $T_{n h}$ was nearly independent of contact angles $\theta_{c}$ when $\theta_{c}<\theta_{c r}$ and $\theta_{c} \geq 180^{\circ}-\theta_{c r}$. However, it decreased rapidly with increasing contact angle beyond the critical angle and featured a minimum at $90^{\circ}$ before increasing rapidly again for contact angle between $90^{\circ}$ and $180^{\circ}-\theta_{c r}$. In other words, four distinct optical regimes can be defined with respect to the critical angle $\theta_{c r}$ namely (i) Regime I with $\theta_{c}<\theta_{c r}$, (ii) Regime II with $\theta_{c r} \leq \theta_{c}<90^{\circ}$, (iii)
Regime III with $90^{\circ} \leq \theta_{c}<180^{\circ}-\theta_{c r}$, and (iv) Regime IV with $\theta_{c} \geq$ $180^{\circ}-\theta_{c r}$. Finally, note that droplet with large refractive index resulted in smaller normal-hemispherical transmittance compared with smaller refractive index of droplets, for any contact angles.

Figs. 8(a)-(d) schematically illustrate the effect of droplet contact angle on the normal-hemispherical transmittance of wet windows for the optical Regimes I to IV previously described. For regime I with $\theta_{c}<\theta_{c r}$ [Fig. 8(a)] and regime IV with $\theta_{c} \geq 180^{\circ}-$ $\theta_{c r}$ [Fig. 8(d)], no total internal reflection occurred at the droplet/ air interface so that contact angle had little effect on the normalhemispherical transmittance. However, the normal-hemispherical transmittance $T_{n h}$ was systematically larger in Regime I $\left(\theta_{c}<\theta_{c r}\right)$ than in Regime IV $\left(\theta_{c} \geq 180^{\circ}-\theta_{c r}\right)$. This was due to the fact that, for a given droplet projected surface area coverage, photon bundles passing near the periphery of droplets on hydrophobic surfaces (Regime IV) had to pass through the window/air interface before interacting with the droplet instead of directly through the window/droplet interface. However, the normal transmittance of the window/air interface is smaller than that of the window/droplet interface due to the larger refractive index mismatch. As a result, the normal-hemispherical transmittance for hydrophobic surfaces such that $\theta_{c} \geq 180^{\circ}-\theta_{c r}$ (Regime IV) was smaller than that for hydrophilic surfaces when $\theta_{c}<\theta_{c r}$ (Regime I).

Finally, for Regime II with $\theta_{c r} \leq \theta_{c}<90^{\circ}$ [Fig. 8(b)] and Regime III with $90^{\circ} \leq \theta_{c}<180^{\circ}-\theta_{c r}$ [Fig. 8(c)], total internal reflection occurred when photons passed near the contact line. In fact, in Regime II, the larger the contact angle the more photon bundles were reflected back into the droplets and in the glass 
(a) Regime I: $\theta_{c}<\theta_{c r}$

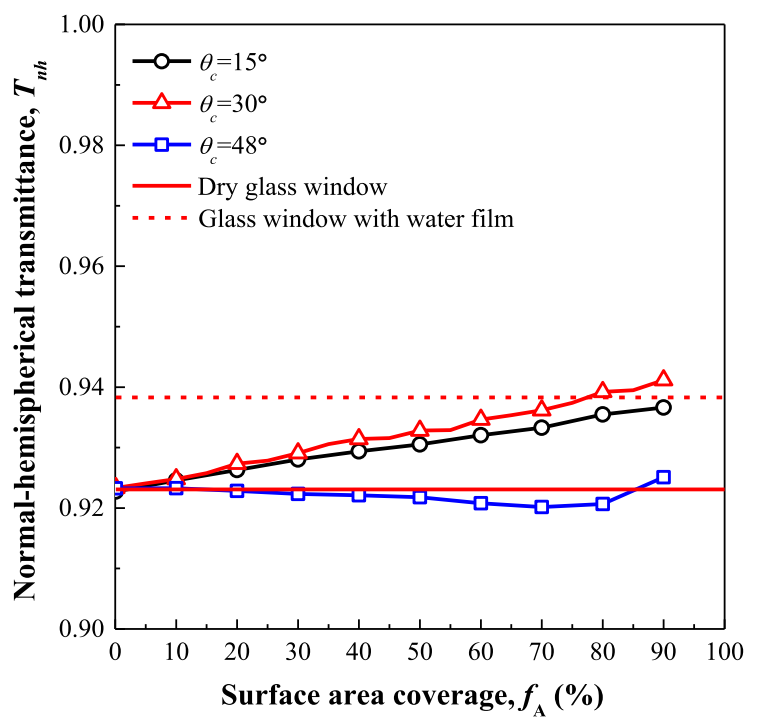

(c) Regime III: $90^{\circ} \leq \theta_{c}<180^{\circ}-\theta_{c r}$

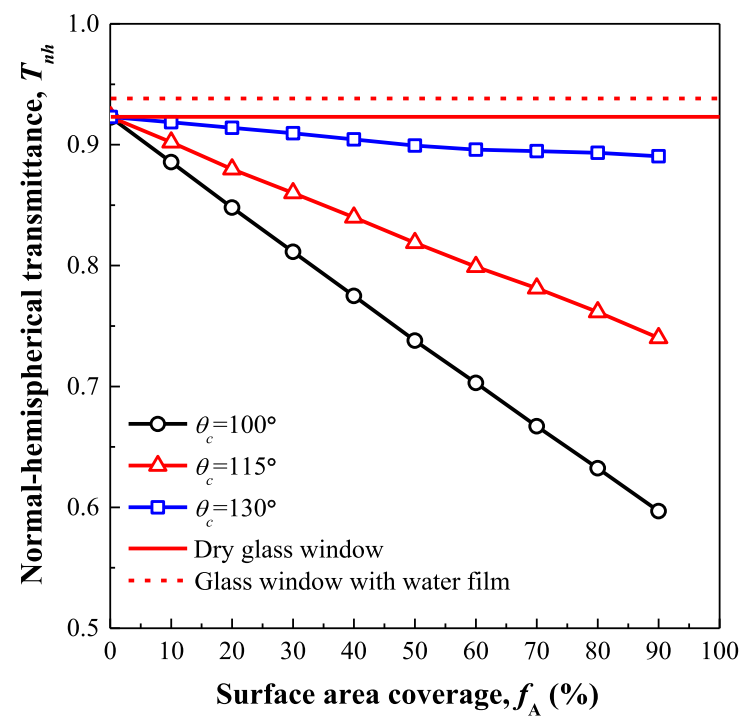

(b) Regime II: $\theta_{c r} \leq \theta_{c}<90^{\circ}$

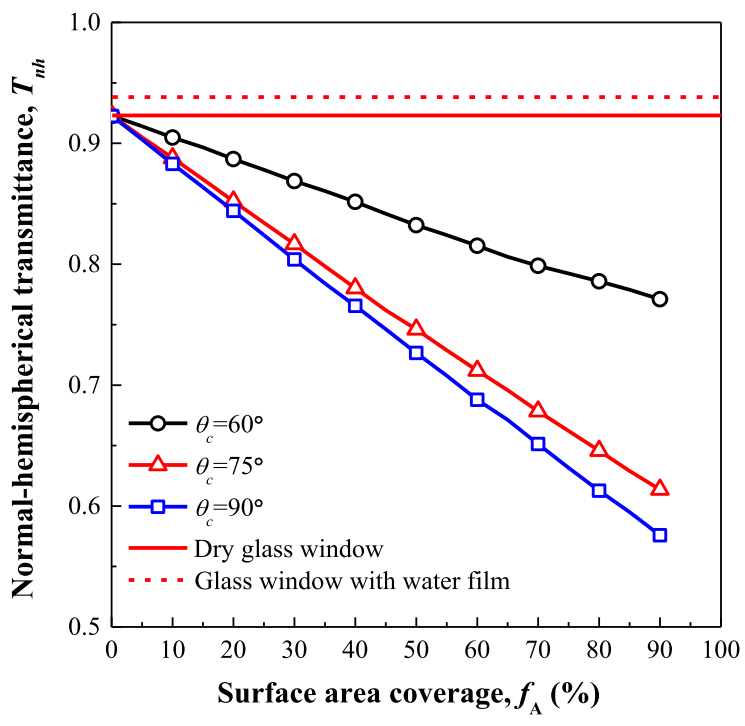

(d) Regime IV: $\theta_{c} \geq 180^{\circ}-\theta_{c r}$

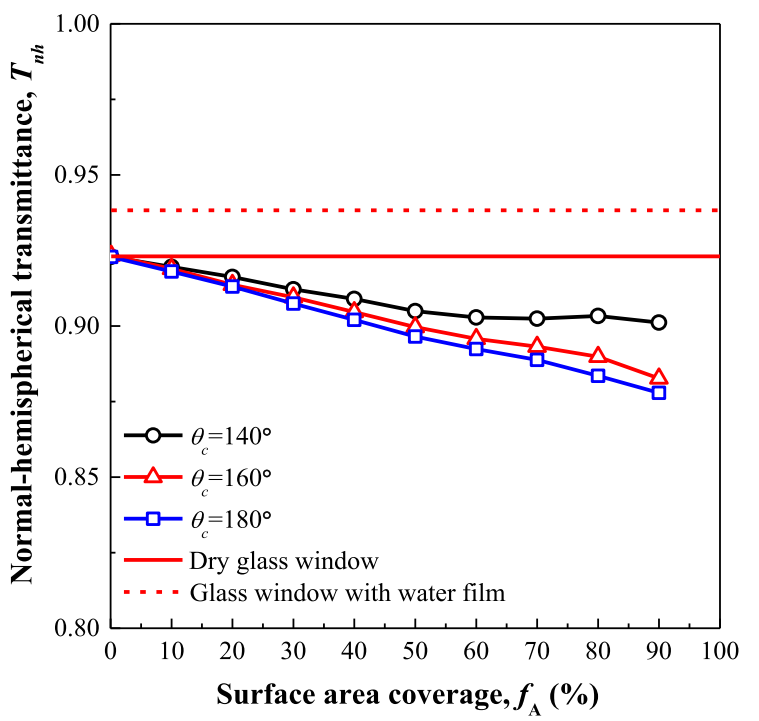

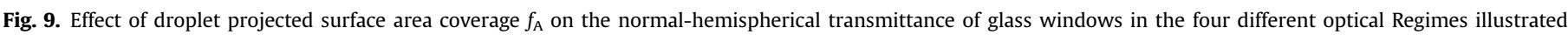
in Fig. 8.

window as total internal reflection became increasingly important and transmittance decreased. On the other hand, in Regime III $\left(90^{\circ}\right.$ $\leq \theta_{c}<180^{\circ}-\theta_{c r}$ ), the larger the contact angle the fewer photon bundles were reflected back to the glass window from the droplet/ air interface and transmittance increased. This can be attributed to the fact that the area of droplet/air interface where total internal reflection occurred decreased as $\theta_{c}$ increased.

\subsection{Effect of projected surface area coverage}

Fig. 9(a)-(d) plot the normal-hemispherical transmittance as a function of projected surface area coverage for different contact angles in the four optical regimes previously identified. In Regime I for $\theta_{c}<\theta_{c r}=48.8^{\circ}$, the normal-hemispherical transmittance of the wet glass windows increased very slightly with increasing droplet projected surface area coverage [Fig. 9(a)]. In other words, the presence of droplets increased slightly the transmittance above that of dry glass window as droplets featured smaller index mismatch with air than glass. These results are consistent with experimental measurements by Geoola et al. [6,7] with auti-drop PE films. Moreover, in some cases (e.g., for $\theta_{c}=30^{\circ}$ and $f_{\mathrm{A}}>85 \%$ ), the normal-hemispherical transmittance exceeded that of transparent glass window covered with a non-absorbing water film of arbitrary thickness denoted by $T_{n h, f}$ and expressed as [32]

$T_{n h f}=\frac{\left(1-\rho_{a w}\right)\left(1-\rho_{w f}\right)\left(1-\rho_{f a}\right)}{\left(1-\rho_{a w} \rho_{w f}\right)\left(1-\rho_{w f} \rho_{f a}\right)-\rho_{a w} \rho_{f a}\left(1-\rho_{w f}\right)^{2}}$

where $\rho_{a w}, \rho_{w f}$, and $\rho_{f a}$ are the specular reflectivities under normal incidence at the air/window, window/film, and film/air interfaces respectively given by $\rho_{i j}=\left(n_{i}-n_{j}\right)^{2} /\left(n_{i}+n_{j}\right)^{2}$. This could be attributed to total internal reflection occurring at the top surface of the glass window, so that incidence radiation was reflected back into the droplets and eventually transmitted, as suggested by Tow [15].

In the other optical Regimes II to IV, the normal-hemispherical transmittance decreased almost linearly with increasing projected surface area coverage and was always smaller than that of dry glass 
window. In Regimes II and III, such that $\theta_{c r} \leq \theta_{c}<180^{\circ}-\theta_{c r}$, this was due to total internal reflection at the droplet/air interface, as illustrated in Fig. 9(b) and (c). In Regime IV, such that $\theta_{c} \geq 180^{\circ}-\theta_{c r}$, the normalhemispherical transmittance decreased linearly albeit only slightly with projected surface area coverage and was nearly independent of contact angle, as previously discussed.

\section{Conclusion}

This study investigated numerically the directional-hemispherical transmittance of transparent windows supporting non-absorbing capshaped water droplets condensing on its backside. It considered not only monodisperse droplets ordered in a hexagonal pattern as studied in the literature but also and for the first time, randomly distributed and polydisperse droplets with contact angle ranging between $0^{\circ}$ (hydrophilic) and $180^{\circ}$ (superhydrophobic) and projected surface area coverage between 0 and $90 \%$. The normal-hemispherical transmittance of these different configurations was predicted using Monte Carlo ray-tracing method. Results indicate that the directional-hemispherical transmittance was independent of droplets' spatial arrangement and size distribution. In fact, it depended only on (i) the incident angle, (ii) the optical properties of the window and droplets, and on (iii) the droplet contact angle and (iv) projected surface area coverage. In all cases, the directional-hemispherical transmittance decreased with increasing incident angle, except for a slight increase when contact angle was $90^{\circ}$. In addition, four optical regimes in the normal-hemispherical transmittance were identified and defined with respect to the critical angle $\theta_{c r}$ for total internal reflection at the droplet/air interface. For droplet contact angle $\theta_{c}$ smaller than the critical angle $\theta_{c r}$ (Regime I), the normal-hemispherical transmittance was nearly independent of contact angle and increased with projected surface area coverage thanks to the smaller refraction index mismatch at the droplet/air interface than at the window/air interface. However, for contact angles beyond $\theta_{c r}$ and smaller than $90^{\circ}$ (Regime II), the normal-hemispherical transmittance decreased rapidly with contact angles while it increased with contact angle increasing from $90^{\circ}$ to $180^{\circ}-\theta_{c r}$ (Regime III). This was due to total internal reflection at the droplet/air interface. For contact angles exceeding $180^{\circ}-\theta_{c r}$ (Regime IV), the normal-hemispherical transmittance was nearly independent of contact angles but slight smaller than that of Regime I. In Regimes II to IV, the normal-hemispherical transmittance decreased linearly with increasing droplet projected surface area coverage. In practice, windows with a hydrophilic back surface, such that $\theta_{c}<\theta_{c r}$, feature increasing transmittance with increasing droplet projected surface area coverage. Such behavior is highly desirable for greenhouses, solar desalination, and photobioreactors systems, to name a few. The second best option in mitigating the effects of condensing droplets on window transmittance consists of windows with superhydrophobic back surface.

\section{Acknowledgments}

This study was supported in part by National Natural Science Foundation of China (No. 51406006) and by the China Scholarship Council (No. 201506025015).

\section{Appendix A. Supporting information}

Supplementary data associated with this article can be found in the online version at http://dx.doi.org/10.1016/j.jqsrt.2017.03.012.

\section{References}

[1] Briscoe BJ, Galvin KP. The effect of surface fog on the transmittance of light. Sol Energy 1991;46:191-197.

[2] Pollet IV, Pieters JG. Condensation and radiation transmittance of greenhouse cladding materials: part 1, laboratory measuring unit and performance. J Agric Eng Res 1999;74:369-377.

[3] Pollet IV, Pieters JG. Condensation and radiation transmittance of greenhouse cladding materials, part 2: results for a complete condensation cycle. J Agric Eng Res 2000;75:65-72.

[4] Pollet IV, Pieters JG. Condensation and radiation transmittance of greenhouse cladding materials, part 3: results for glass plates and plastic films. J Agric Eng Res 2000;77:419-428.

[5] Pollet IV, Pieters JG. PAR transmittances of dry and condensate covered glass and plastic greenhouse cladding. Agric For Meteorol 2002;110:285-298.

[6] Geoola F, Kashti Y, Peiper UM. A model greenhouse for testing the role of condensation, dust and dirt on the solar radiation transmissivity of greenhouse cladding materials. J Agric Eng Res 1998;71:339-346.

[7] Geoola F, Kashti Y, Levi A, Brickman R. Quality evaluation of anti-drop properties of greenhouse cladding materials. Polym Test 2004;23:755-761.

[8] Cemek B, Demir Y. Testing of the condensation characteristics and light transmissions of different plastic film covering materials. Polym Test 2005;24:284-289.

[9] Hsieh C, Rajvanshi AK. The effect of dropwise condensation on glass solar properties. Sol Energy 1977;19:389-393.

[10] Pieters JG, Deltour J, Debruyckere M. Light transmission through condensation on glass and polyethylene. Agric For Meteorol 1997:85:51-62.

[11] Pieters JG. Interaction effects in simulating the light transmission through condensation drops on greenhouse covers. Trans ASAE 1997;40:1463-1465.

[12] Farkas I, Weihs P, Biro A, Laube W, Eitzinger J, Wojcicki A. Modelling of radiative PAR transfer in a tunnel greenhouse. Math Comput Simul 2001;56:357-368.

[13] Soriano T, Montero JI, Sánchez-Guerrero MC, Medrano E, Antón A, Hernández $\mathrm{J}$, et al. A study of direct solar radiation transmission in asymmetrical multispan greenhouses using scale models and simulation models. Biosyst Eng 2004:88:243-253.

[14] Max JFJ, Reisinger G, Hofmann T, Hinken J, Tantau H-J, Ulbrich A, et al. Glassfilm-combination: opto-physical properties and energy saving potential of a novel greenhouse glazing system. Energy Build 2012;50:298-307.

[15] Tow EW. The antireflective potential of dropwise condensation. J Opt Soc Am A Opt Image Sci Vision 2014;31:493-499.

[16] Heinemann PH, Walker PN. Effects of greenhouse surface heating water on light transmission. Trans ASAE 1987;30:215-220.

[17] Jaffrin A, Morisot A. Mechanism of light transmission through wet polymer films. Acta Hortic 1990;281:11-24.

[18] Jaffrin A, Urban L. Optimization of light transmission in modern greenhouses. Acta Hortic 1990;281:25-33.

[19] Pieters JG, De Clercq S. Pollet IV. Simulating the effect of condensation type on greenhouse shortwave radiation transmission and utilization. Acta Hortic 1998:456:313-320.

[20] Yaka Çetin F, İpekoğlu B. Impact of transparency in the design of protective structures for conservation of archaeological remains. J Cult Herit 2013;14:2124.

[21] Chaibi MT, Jilar T. Effects of a solar desalination module integrated in a greenhouse roof on light transmission and crop growth. Biosyst Eng 2005;90:319-330.

[22] Pruvost J, Leborgne F, Artu A, Cornet JF, Legrand J. Industrial photobioreactors and scale-up concepts, in "Photobioreaction Engineering". Special Issue of Advances Chemical Engineering; 48; 2016.

[23] Vincent R. A theoretical analysis and computer simulation of the growth of epitaxial films. Proc R Soc Lond A 1971;321:53-68.

[24] Rose JW, Glicksman LR. Dropwise condensation- the distribution of drop sizes. Int J Heat Mass Trans 1973:16:411-425.

[25] Beysens D, Knobler CM. Growth of breath figures. Phys Rev Lett 1986;57:14331436.

[26] Rubin M. Optical properties of soda lime silica glasses. Sol Energy Mater $1985 ; 12: 275-288$.

[27] Hale GM, Querry MR. Optical constants of water in the 200-nm to 200- $\mu \mathrm{m}$ wavelength region. Appl Opt 1973;12:555-563.

[28] De Gennes PG, Brochard-Wyart F, Quéré D. Capillarity and wetting phenomena: drops, bubbles, pearls, waves. Springer Science \& Business Media; 2013.

[29] Modest MF. Radiative heat transfer.San Diego, CA: Academic Press; 2003.

[30] Kavehpour HP, Ovryn B, McKinley GH. Microscopic and macroscopic structure of the precursor layer in spreading viscous drops. Phys Rev Lett 2003:91:196104.

[31] Hoang A, Kavehpour HP. Dynamics of nanoscale precursor film near a moving contact line of spreading drops. Phys Rev Lett 2011;106:254501.

[32] Howell JR, Mengüç MP, Siegel R. Thermal radiation heat transfer. 5th ed.. Boca Raton: CRC Press; 2015. 